INPLASY

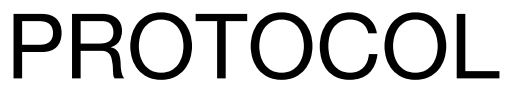

To cite: Ding et al. Metaanalysis on Effectiveness of Shengxuexiaoban Capsules Combined with Hormone

Therapy for Immune Thrombocytopenia. Inplasy protocol 202190031. doi: 10.37766/inplasy2021.9.0031

Received: 11 September 2021

Published: 11 September 2021

Corresponding author: Meng Yang

m13526587119@126.com

Author Affiliation:

The First Affiliated Hospital of Henan University of Traditional Chinese Medicine.

Support: None.

Review Stage at time of this submission: Data extraction.

Conflicts of interest:

None declared.

\section{Meta-analysis on Effectiveness of Shengxuexiaoban Capsules Combined with Hormone Therapy for Immune Thrombocytopenia}

Ding, $\mathrm{M}^{1} ; \mathrm{Li}, \mathrm{B}^{2} ;$ Yang, $\mathrm{M}^{3}$.

Review question / Objective: The main objective of this study is to evaluate effectiveness of the Shengxuexiaoban Capsules combined with hormone therapy for immune thrombocytopenia (ITP) through the Meta analysis.

Condition being studied: Immune thrombocytopenia (ITP) is a common bleeding disorder encountered in clinical practice. It was once named "idiopathic thrombocytopenic purpura", which commonly occurred in both children and adults. ITP is mainly manifested with spontaneous bleeding of the skin and mucous membranes, a decrease in the number of platelets, a prolonged bleeding time[1] etc. At present, glucocorticoids remains the first choice for the treatment of ITP[2,3], which significantly increases the number of platelets in the short term, but its long-term therapeutic effect is not ideal because the medicine causes a series of adverse effects[4], such as central obesity, osteoporosis etc. Studies have discovered that the Shengxuexiaoban Capsules is effective for ITP, because it contains Indigo Naturalis, Cortex Moutan, Forsythia, Agrimonia, Licorice, which are able to clear away heat and toxic materials, cool blood, arrest bleeding, disperse blood stasis and reduce freckles. However, the sample sizes are too small to provide sufficient proofs for evidence-based medicine. In this case, the Meta-analysis was performed on the included RCTs so as to present effective evidences for the clinical treatment.

INPLASY registration number: This protocol was registered with the International Platform of Registered Systematic Review and Meta-Analysis Protocols (INPLASY) on 11 September 2021 and was last updated on 11 September 2021 (registration number INPLASY202190031).

\section{INTRODUCTION}

Review question / Objective: The main objective of this study is to evaluate effectiveness of the Shengxuexiaoban Capsules combined with hormone therapy for immune thrombocytopenia (ITP) through the Meta analysis. 
Condition being studied: Immune thrombocytopenia (ITP) is a common bleeding disorder encountered in clinical practice. It was once named "idiopathic thrombocytopenic purpura", which commonly occurred in both children and adults. ITP is mainly manifested with spontaneous bleeding of the skin and mucous membranes, a decrease in the number of platelets, a prolonged bleeding time[1] etc. At present, glucocorticoids remains the first choice for the treatment of ITP[2,3], which significantly increases the number of platelets in the short term, but its long-term therapeutic effect is not ideal because the medicine causes a series of adverse effects[4], such as central obesity, osteoporosis etc. Studies have discovered that the Shengxuexiaoban Capsules is effective for ITP, because it contains Indigo Naturalis, Cortex Moutan, Forsythia, Agrimonia, Licorice, which are able to clear away heat and toxic materials, cool blood, arrest bleeding, disperse blood stasis and reduce freckles. However, the sample sizes are too small to provide sufficient proofs for evidence-based medicine. In this case, the Meta-analysis was performed on the included RCTs so as to present effective evidences for the clinical treatment.

\section{METHODS}

Participant or population: Patients who were diagnosed with ITP and without limitations in age, gender and race.

Intervention: Hormone therapy combined with the Shengxuexiaoban Capsules.

Comparator: Hormone therapy alone.

Study designs to be included: Randomized controlled trials.

Eligibility criteria: Patients who were diagnosed with ITP and without limitations in age, gender and race.

Information sources: A full-test search was performed in the databases to select the RCTs where the hormone therapy was combined with the Shengxuexiaoban Capsules to treat ITP, including China
National Knowledge Infrastructure (CNKI), VIP database (VIP), China Biology Medicine disc (CBMdisc), Wanfang Data Knowledge Service Platform (Wanfang Da-ta), PubMed and Embase. The retrieval time was from the establishment of the database to April 21st, 2021, and the Chinese keywords mainly included"升血小板胶囊 (Shengxuexiaoban Capsules)", 免疫性血小板 减少症(Immune Thrombocytopenia)" and "激素(Hormone)", and the English keywords included "Immune Thrombocytopenia", "Idiopathic Thrombocytopenia", "shengxuexiaobanjiaonang" and "Glucocorticoid". The search mode of keywords combined with free words was adopted.

Main outcome(s): Total Effective Rate; Recurrence Rate; Number of Platelets; Recovery Time of Platelets.

Quality assessment / Risk of bias analysis: The quality of the included RCTs was evaluated by the risk of bias assessment tool of Cochrane Handbook for Systematic Reviews of Interventions (Version 5.1.0). The biases mainly covered six aspects, namely, selection bias, performance bias, detection bias, attrition bias, reporting bias and other biases, and were categorized with "low risk", "unclear risk" and "high risk" one by one.

Strategy of data synthesis: In this study, RevMan5.3 and Stata16.0 software were adopted for the Meta-analysis. The $P$ and 12 values were used to assess the heterogeneity.

\section{Subgroup analysis: None.}

Sensitivity analysis: When $P$ value $<0.1$ or $12>50 \%$, the heterogeneity appeared and a random-effect model was applied for heterogeneity and sensitivity analysis, while $P$ value $>0.1$ or $12<50 \%$, a fixedmodel was applied.

Language: No restriction.

Country(ies) involved: China. 
Keywords: Shengxuexiaoban Capsules;

I m m une Thrombocytopenia;

Glucocorticoid; Meta-analysis.

Contributions of each author:

Author 1 - Mengyuan Ding.

Author 2 - Bing Li.

Author 3 - Meng Yang. 